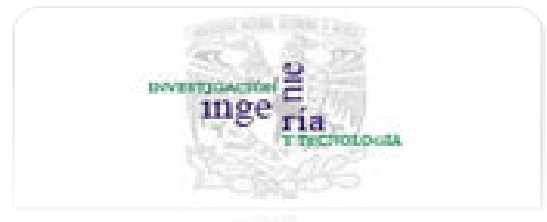

Ingeniería. Investigación y Tecnología

ISSN: $1405-7743$

iit.revista@gmail.com

Universidad Nacional Autónoma de México

México

Fuentes-Silva, C.; Noriega-Ponce, A.; Ocampo-Mortera, M.A.; Serroukh, I.

Control neuronal auto-ajustable en tiempo real de un proceso de producción de fibra óptica polimérica

Ingeniería. Investigación y Tecnología, vol. XIII, núm. 2, abril-junio, 2012, pp. 175-184

Universidad Nacional Autónoma de México

Distrito Federal, México

Disponible en: http://www.redalyc.org/articulo.oa?id=40423274005

Cómo citar el artículo

- Número completo

- Más información del artículo

Página de la revista en redalyc.org

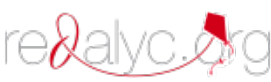

Sistema de Información Científica

Red de Revistas Científicas de América Latina, el Caribe, España y Portugal

Proyecto académico sin fines de lucro, desarrollado bajo la iniciativa de acceso abierto 


\title{
Control neuronal auto-ajustable en tiempo real de un proceso de producción de fibra óptica polimérica
}

\author{
Real Time Self-Adjusting Neuro Control of a Polymeric \\ Optical Fiber Production Process
}

\author{
Fuentes-Silva C. \\ Universidad Autónoma de Querétaro, México \\ Correo:ingcfsqro@hotmail.com \\ Noriega-Ponce A. \\ Universidad Autónoma de Querétaro, México \\ Correo:anoriega@uaq.mx
}

\author{
Ocampo-Mortera M.A. \\ Centro de Física Aplicada y Tecnología Avanzada, UNAM \\ Campus Juriquilla, Querétaro, México \\ Correo:oca@fata.unam.mx \\ Serroukh I. \\ Universidad Autónoma de Querétaro, México \\ Correo:ibraser@hotmail.com
}

Información del artículo: recibido: enero de 2010, reevaluado: octubre de 2010, aceptado: abril de 2011

\section{Resumen}

En este trabajo se presenta un control neuronal auto ajustable en tiempo real para la fabricación de fibras ópticas poliméricas mediante el estirado de preformas, enfocado a controlar las variaciones de baja frecuencia del diámetro de las fibras producidas. Aunque el estirado de fibras ópticas es un proceso de variables extendidas, y el sistema propuesto sólo emplea 7 neuronas con parámetros ajustables, los resultados obtenidos muestran la posibilidad de reducir la máxima variación de diámetro en $50 \%$, respecto a la observada cuando no se emplea un control del proceso y el diámetro de las fibras obtenidas se mantiene con variaciones menores a $4 \%$ del diámetro promedio, con lo que se pueden cumplir las especificaciones comerciales y normativas internacionales.

\section{Abstract}

A real time self-adjusting neuro control scheme for optical fiber drawing is presented in this paper. The fiber presents high and low frequency variations in the obtained diameter, being these last ones caused by variations in the preform diameter and the distribution of the molecular weight which affects the rheologic behavior during the drawing process of the material. In order to control these variations an algorithm of self-adjusting control based on a three layers perceptron type neural network is proposed.

\section{Descriptores}

- fibra óptica polimérica

- red neuronal

- control autoajustable

\section{Keywords}

- optic fiber

- polymeric neural network

- self-tuning control 


\section{Introducción}

La obtención de fibras ópticas poliméricas mediante el estirado de preformas es un proceso no-lineal, cuyo comportamiento obedece a una dinámica de parámetros continuamente distribuidos. Los modelos matemáticos para representar su comportamiento son complejos, requieren la solución de las ecuaciones de balance de masa y de energía en una región de dimensiones considerables y, salvo por la ecuación que representa la conservación global de masa bajo condiciones estacionarias, no pueden ser sustituidos por expresiones representativas suficientemente simples para incorporarse en un sistema de control convencional (Reeve et al., 2003). Debido a esto, el control del proceso requiere de herramientas eficientes con capacidad adaptativa para representar la dinámica del sistema bajo condiciones que varían de forma impredecible. En el presente trabajo exploramos la utilidad de las redes neuronales para abordar este problema (Colmenares et al., 2001).

El estirado de fibras ópticas, principalmente de óxido de silicio, ha sido abordado con múltiples enfoques. Desde 1978, Nakahara propuso que al estudiar los efectos de diferentes condiciones térmicas y mecánicas sobre la estabilidad en el diámetro de las fibras producidas y sobre su resistencia a la tensión, las variaciones de temperatura en el horno son el principal factor que afecta las variaciones de alta frecuencia en el diámetro de la fibra. También sugirió que las variaciones de baja frecuencia son ocasionadas por variaciones longitudinales en el diámetro de la preforma y propuso un control basado en el ajuste retroalimentado de la velocidad de estirado para reducir estas últimas.

Otros autores han propuesto el control de la temperatura de los gases alimentados al horno de estirado, mediante el control de la proporción de gas frío y caliente introducida al proceso. Bajo este esquema se pueden compensar las variaciones de diámetro de mediana frecuencia de la fibra producida mediante el enfriamiento local del cuello de estirado, aunque se ve afectada su resistencia mecánica por el almacenamiento de esfuerzos producidos por una mayor velocidad de enfriamiento del material (Imoto et al., 1989).

En el caso de las fibras ópticas poliméricas, las variaciones locales del peso molecular manifiestan su impacto tanto en el comportamiento reológico durante el proceso de estirado del material, como en las características mecánicas y dimensionales de la fibra producida (Valenzuela, 2006). El control del proceso se ve complicado por la mayor variabilidad de las características termomecánicas del material. Adicionalmente, el mayor diámetro típico de las fibras poliméricas introduce una mayor velocidad del flujo de material con una consecuente mayor inestabilidad del proceso.

Como ya se ha mencionado, en el presente trabajo se aborda el problema de controlar el diámetro de fibras ópticas de Poli-metil-metacrilato (PMMA) producidas mediante el estirado de preformas, empleando un sistema que retroalimenta la velocidad de extracción de la fibra producida y usa como controlador una red neuronal de tres capas tipo perceptrón, que ajusta sus parámetros en cada ciclo de operación, en tiempo real firme (Noriega et al., 2004). El sistema de control se desarrolló en una plataforma LabVIEW, sobre la que se integró la instrumentación de la torre de estirado empleada para monitorear el diámetro de la fibra y para efectuar los ajustes necesarios en la velocidad de estirado. Los resultados son comparados con los obtenidos mediante un sistema que fija los parámetros de proceso en los valores necesarios para producir una fibra con las características deseadas. El esquema de control propuesto tiene la capacidad de retropropagar el error de regulación en lugar del error de salida de la red, por lo que es posible sustituir los coeficientes de la red neuronal obtenidos durante el entrenamiento previo de la red por los valores generados por un proceso de adaptación subsecuente en tiempo real.

\section{Descripción del sistema de estirado de preformas y del modelo de proceso. Planteamiento experi- mental}

\section{Descripción de la torre de estirado}

La torre de estirado consta de un subsistema para la alimentación de la preforma, de un subsistema para la extracción de la fibra estirada, de un horno tubular de dos zonas para el reblandecimiento de la preforma con obturadores en ambos extremos para limitar el flujo convectivo de aire entre la cámara de calentamiento y el exterior, y de un monitor ZUMBACH XY18, que mide el diámetro de la fibra producida con una precisión de \pm 1 micra. Los sistemas de alimentación de la preforma y de extracción de la fibra se encuentran conectados a un controlador de movimiento de dos ejes, GALIL DMC1425, que controla la velocidad de los motores correspondientes con estabilidades de $\pm 0.001 \mathrm{rev} / \mathrm{min}$ y de $\pm 0.01 \mathrm{rev} / \mathrm{min}$, respectivamente. Las dos secciones del horno son controladas mediante controladores independientes de temperatura OMRON E5EN tipo PID, con una estabilidad de $\pm 0.1^{\circ} \mathrm{C}$ en la zona superior y de $\pm 0.2^{\circ} \mathrm{C}$ en la zona inferior. Estos subsistemas se encuentran comunicados mediante conexiones RS-232 y Ethernet con una PC de control central que establece los 
parámetros de proceso mediante el programa LabVIEW desarrollado. La figura 1 muestra en forma esquemática el diagrama de la torre de estirado y la figura 2 muestra una foto del sistema empleado en este trabajo, desarrollado en el CFATA-UNAM.

La operación rutinaria de la torre de estirado inicia calentando cada zona del horno de reblandecimiento a las temperaturas establecidas. Una vez estabilizada la temperatura del horno se instala la preforma en el sistema de alimentación y se inicia su descenso a la velocidad establecida para el proceso. En algún momento, después de introducida la preforma al horno, ésta alcanza su temperatura de reblandecimiento y empieza a fluir por gravedad. Se forma así un cuello de estirado que se alarga hasta que el extremo inferior de la preforma sale por el extremo inferior del horno. Se corta la parte gruesa del material extraído y se instala la fibra obtenida en el sistema de extracción de fibra, que la sigue jalando mientras dura el proceso. Durante la etapa inicial del estirado, la fibra obtenida se mantiene cambiando su diámetro hasta que se estabiliza el proceso, después de lo cual, las variaciones de diámetro observadas corresponden a las perturbaciones inherentes que sufre el proceso. El control desarrollado modifica la velocidad de estirado para controlar las variaciones de diámetro de la fibra mediante el algoritmo de control alimentado al programa, que toma como entrada los valores de diámetro reportados por el monitor de diámetro.

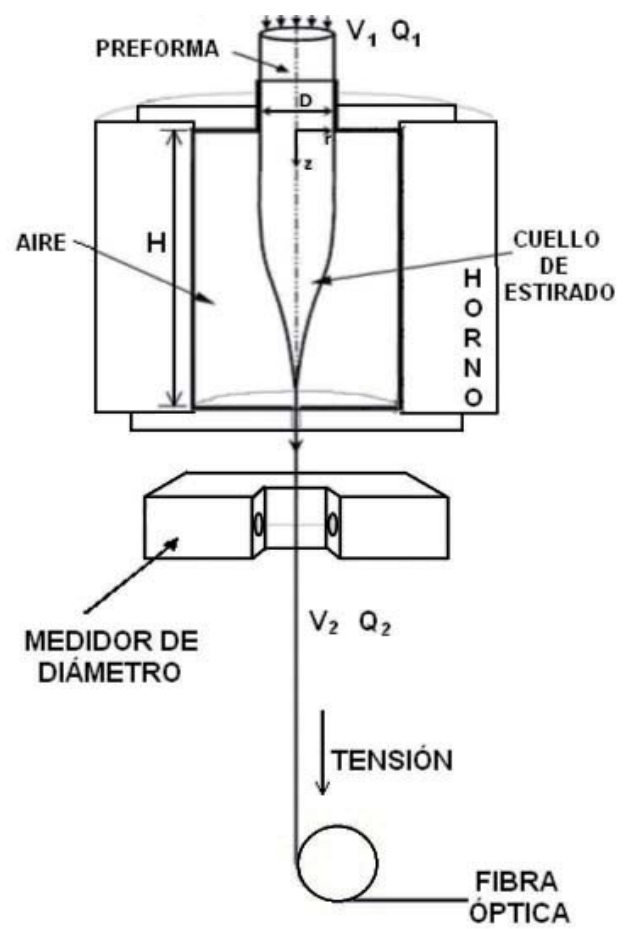

Figura 1. Esquema de la torre de estirado

\section{Modelos del proceso}

La descripción matemática del flujo de los materiales que constituyen una preforma durante el proceso de obtención de una fibra óptica ha sido modelada, entre otros, por Reeve et al. (2003) y Lee et al. (2006), mediante modelos genéricos que consideran los flujos radiativo y convectivo de calor entre la superficie interna del horno y la preforma, y los concomitantes flujos de calor por conducción y masa en el interior de la preforma. La aproximada simetría cilíndrica del problema permite reducir el número de variables a determinar como una función de la posición y del tiempo, de manera que sólo las componentes radial y axial de los flujos correspondientes son introducidas en los modelos.

Las ecuaciones que gobiernan los flujos de masa, momento y energía, se pueden escribir en coordenadas cilíndricas de la siguiente manera:

Conservación de la masa:

$\frac{\partial \rho}{\partial t}+\frac{1}{r} \frac{\partial}{\partial r}(r \rho u)+\frac{\partial}{\partial z}(\rho v)=0$

Conservación del momento:

$\rho \frac{D v}{D t}=\frac{1}{r} \frac{\partial}{\partial r}\left(r \sigma_{r r}\right)+\frac{\partial \sigma_{r z}}{\partial z}-2 \mu \frac{u}{r^{2}}, \mathrm{y}$

$\rho \frac{D v}{D t}=\frac{1}{r} \frac{\partial}{\partial r}\left(r \sigma_{r z}\right)+\frac{\partial \sigma}{\partial z}+\rho g$

Flujo de calor:

$\rho c_{P} \frac{D T}{D t}=\frac{1}{r} \frac{\partial}{\partial r}\left(k_{m} r \frac{\partial T}{\partial r}\right)+\frac{\partial}{\partial z}\left(k_{m} \frac{\partial T}{\partial z}\right)+W_{i}+\mu \Phi$.

Donde:

$$
\begin{aligned}
& \frac{D}{D t}=\frac{\partial}{\partial t}+u \frac{\partial}{\partial r}+v \frac{\partial}{\partial z}, \sigma_{r r}=-P+2 \mu \frac{\partial u}{\partial r}, \\
& \sigma_{z z}=-P+2 \mu \frac{\partial v}{\partial z}, \sigma_{r z}=2 \mu\left(\frac{\partial u}{\partial z}+\frac{\partial v}{\partial r}\right), \mathrm{y} \\
& \Phi=2\left[\left(\frac{\partial u}{\partial r}\right)^{2}+\left(\frac{u}{r}\right)^{2}+\left(\frac{\partial v}{\partial z}\right)^{2}\right]+\left(\frac{\partial u}{\partial z}+\frac{\partial v}{\partial r}\right) .
\end{aligned}
$$


Las variables a determinar como una función de la posición y del tiempo son la presión, $P$, la temperatura, $T$, y las componentes radial y axial de la velocidad, $u$ y $v$, respectivamente. $\rho$ es la viscosidad, $\mu$ es la viscosidad, $k_{m}$ es la conductividad térmica y $C_{p}$ es el calor específico del fluido en consideración (aire o material de la preforma); $g$ es la aceleración de la gravedad, y $W_{i}$ es la generación de calor por unidad de tiempo y de volumen.

Las condiciones en la frontera deben considerar la distribución de temperaturas en la superficie cilíndrica del horno y sus tapas, y los modelos para las correspondientes emisión radiativa y transferencia convectiva. Asimismo, se deben incluir las distribuciones de temperatura en la sección transversal de la preforma entrante al horno y de la fibra saliente, así como sus velocidades. Comúnmente, la velocidad de salida de la fibra no coincide con la velocidad de extracción, pues ésta aun no se ha consolidado a la salida del horno. La superficie de la preforma, desde el punto de introducción hasta su salida como fibra, pasando por el cuello de estirado, es una variable del proceso que debe ser determinada de manera autoconsistente con la solución de las ecuaciones de conservación planteadas.

Dado que la viscosidad del material de la preforma es fuertemente dependiente de la temperatura, llegando a tener variaciones de $10 \%$ con un grado de variación en la temperatura bajo las condiciones normales de procesamiento, el flujo del material para formar la fibra se encuentra fuertemente acoplado con el flujo térmico en todo el sistema, incluso con la historia térmica de la preforma, que además de depender de la manera en que se realiza su calentamiento inicial, es calentada por el flujo convectivo en el exterior del horno antes de ingresar.

Siendo la principal contribución al flujo de calor entre el horno y la superficie de la preforma el flujo radiativo, las dificultades para evaluar los factores dependientes de la orientación de las superficies y las respuestas espectrales dependientes de la absorción y emisión en las superficies relevantes, complican aún más el cálculo de las soluciones buscadas.

Las soluciones a las ecuaciones de conservación presentadas para determinar el flujo de materiales durante el estirado de una preforma se han obtenido numéricamente y comparado con observaciones experimentales. En el caso de las fibras de óxido de silicio, las diferencias prevalecientes entre los modelos y lo observado muestran a las variaciones de diámetro de alta frecuencia como un comportamiento difícil de modelar, ya que éstas son consecuencia del establecimiento de regímenes oscilatorios complejos en las corrientes convectivas alrededor del cuello de estirado que promueven cambios rápidos de la viscosidad del material en las regiones de formación final de las fibras. De igual importancia son las diferencias dimensionales de la preforma con las predicciones del modelo, que aunque en las regiones de baja deformación se mantienen por debajo de 5\%, en la región de formación de la fibra pueden alcanzar diferencias cercanas a $100 \%$.

En el caso del estirado de fibras ópticas poliméricas, el modelado del proceso presenta problemas adicionales, debido a las inhomogeneidades del peso molecular de los materiales que conforman a las preformas, que pueden ocurrir con variaciones del orden de $5 \%$ o mayores, a lo largo del eje de la preforma en materiales convencionales (García et al., 2010). Tales irregularidades en los materiales se manifiestan comúnmente como variaciones del diámetro de frecuencias intermedias y bajas, que pueden tornar incontrolable el proceso si se desea aumentar la velocidad de producción, ya que en estas condiciones el cuello de estirado tiende a aumentar en longitud, pudiendo contener regiones de diferente fluidez, difícilmente controlables por los parámetros de proceso comúnmente disponibles para tal efecto.

Independientemente de las dificultades para resolver los problemas involucrados en el modelado del proceso de estirado, la descripción de algunos aspectos generales del estado estacionario son importantes para establecer las condiciones de referencia sobre la que se pueden identificar las variables que juegan un papel central en el control del proceso.

Una vez que se ha formado el cuello de estirado y que el proceso ha evolucionado por un tiempo sin modificaciones en la temperatura del horno y en las velocidades de alimentación de la preforma, $V_{1}$, y de extracción de la fibra, $V_{2}$, el sistema alcanza, en términos generales y de manera natural, un estado estacionario en el que el flujo del material de la preforma a la entrada del horno compensa su salida en forma de fibra en el extremo inferior del horno, reflejando el balance de masa en tales condiciones.

Refiriéndonos a la figura 1, que representa de manera esquemática el proceso estudiado, los flujos de masa de la preforma en la entrada del horno, $Q_{1}$, y de la fibra a la salida obtenida, $Q_{2}$, están dados por:

$Q_{1}=\pi D^{2} V_{1} \rho_{1} / 4$ y $Q_{2}=\pi d^{2} V_{2} \rho_{2} / 4$

donde $D$ y $d$ son los diámetros de la preforma y de la fibra, respectivamente, y $\rho_{1}, \rho_{2}$ son las densidades correspondientes. En estado estacionario, y considerando que no hay diferencia significativa en la densidad del polímero a la entrada contra la densidad del mismo polímero a la salida del horno, tenemos que la velocidad 
de salida de la fibra $\left(\mathrm{V}_{2}\right)$ está relacionada con la velocidad de alimentación $\left(\mathrm{V}_{1}\right)$ de la preforma de la siguiente manera:

$V_{2}=\left(\frac{D}{d}\right)^{2} V_{1}$

Para lograr un estado estacionario en el proceso de estirado deben equilibrarse las transferencias de calor y de masa en cada punto del interior del horno. En el estirado real de una fibra polimérica se tienen múltiples fuentes de fluctuación de tales variables, por lo que el estado estacionario sólo se consigue de manera aproximada. El control del proceso requiere así que las desviaciones observadas sean corregidas mediante esquemas que consideren sus principales causas y tomen en cuenta los complejos comportamientos del sistema.

Considerando que la estabilidad de los movimientos controlados de la preforma y de la fibra, así como de la temperatura de las paredes del horno es bastante buena, los principales factores a considerar para controlar el proceso son las heterogeneidades de la fluidez del material de la preforma, asociadas con distribuciones irregulares del peso molecular, la alta sensibilidad del flujo de calor a los cambios dimensionales de la preforma, la presencia de celdas de convección alrededor del cuello de estirado y en la zona de consolidación de la fibra producida (Reeve et al., 2003). Estas dificultades y el carácter no-lineal de las ecuaciones que determinan el flujo de los materiales confieren una gran dificultad a la obtención de una descripción simple del sistema que permita representar el proceso por expresiones matemáticas cerradas para simplificar los algoritmos de control.

En este trabajo, nos hemos enfocado a minimizar las variaciones de diámetro de baja frecuencia, de particular importancia en el estirado de fibras ópticas poliméricas, pues limitan la posibilidad de cumplir con la normatividad existente y con los estándares establecidos. Para ello, se decidió explorar la habilidad de un controlador neuronal auto-ajustable para controlar el proceso mediante la modificación de la velocidad de estirado en respuesta a las fluctuaciones de diámetro monitoreadas en línea.

\section{Planteamiento experimental}

Para evaluar el sistema de control estudiado, se estiraron 10 preformas de PMMA, de $25.0 \mathrm{~mm}$ de diámetro y aproximadamente $30 \mathrm{~cm}$ de longitud. Se ajustaron las condiciones de proceso para obtener fibras de ópticas de 500 micras de diámetro. En todos los casos, las pre- formas se alimentaron al horno con una velocidad de $1.5 \mathrm{~mm} / \mathrm{min}$. La velocidad de extracción de la fibra se alternó entre dos regímenes; uno de velocidad constante, a $3.75 \mathrm{~m} / \mathrm{min}$, apropiado para obtener el diámetro de fibra deseado, y otro en el que la velocidad fue determinada por el control neuronal a partir de los diámetros monitoreados en tiempo real. Para los parámetros escogidos, el control limitaba la velocidad de producción entre 2.6 y $5.8 \mathrm{~m} / \mathrm{min}$. Las fluctuaciones de diámetro obtenidas fueron analizadas para determinar el grado de control alcanzado.

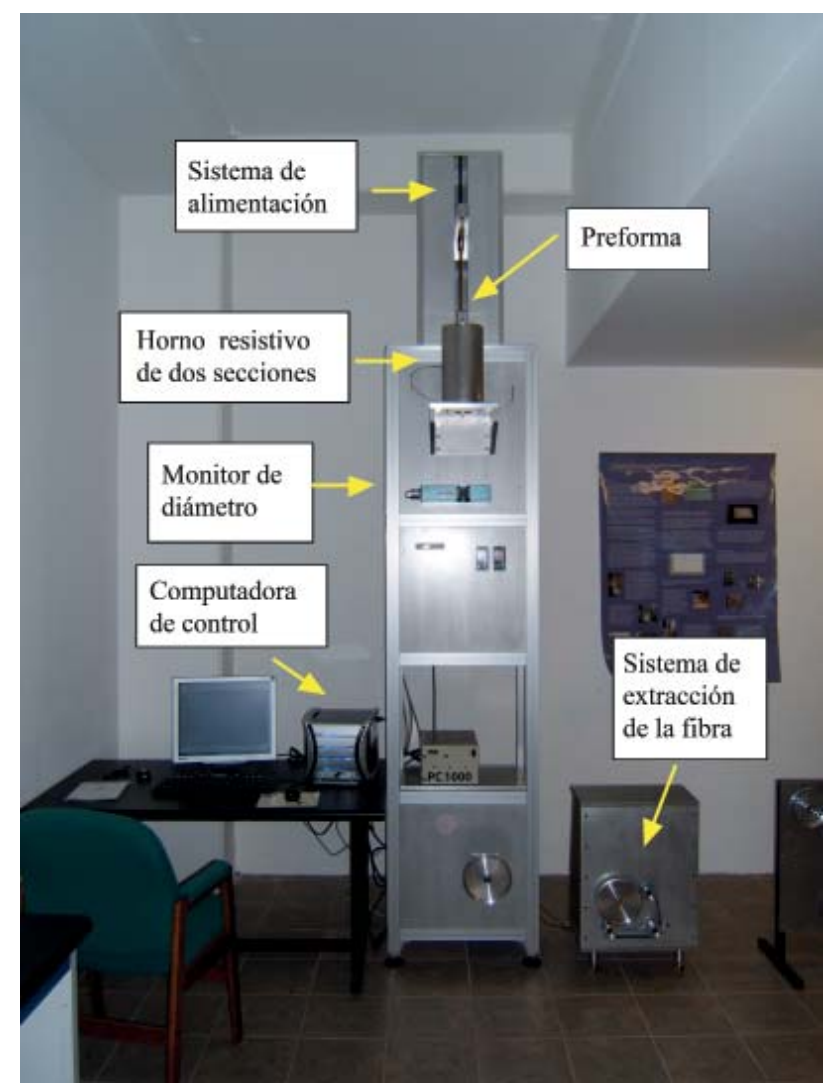

Figura 2. Torre de estirado

\section{Implantación del controlador neuronal auto- ajustable}

Selección del periodo de muestreo

Debido a que las variaciones del diámetro son desconocidas en alta y baja frecuencia, la frecuencia de muestreo se escogió de $4 \mathrm{~Hz}(250 \mathrm{~ms})$ para guardar un margen de seguridad, limitado por la frecuencia de medición del ZUMBACH XY18, el cual garantiza una frecuencia máxima de medición de $5 \mathrm{~Hz}$. Aún cuando el comportamiento es diferente en distintos periodos de muestreo 
las variaciones están determinadas principalmente por las variaciones del peso molecular y las variaciones de temperatura ocasionadas por el comportamiento caótico del flujo dentro del horno, las cuales no pueden ser registradas durante la prueba.

\section{Filtrado del diámetro}

Ya que el objetivo es controlar las variaciones de baja frecuencia modificando la velocidad de estirado, se implementó un filtro de la media móvil que produce el promedio de un número de puntos de la señal de entrada para producir cada punto de la señal de salida:

$y[i]=\frac{1}{M} \sum_{j=0}^{M-1} x[i+j]$

Donde $x$ es la señal de entrada, $y$ la señal de salida y $M$ es el número de puntos empleados para calcular el valor medio (Smith, 1999). Al aplicar el filtro de media móvil se tiene un compromiso entre la eficiencia del filtro para eliminar las altas frecuencias y el retardo generado en la señal filtrada con respecto a la original, como se aprecia en la figura 3, lo cual dificulta el control del sistema. En nuestros experimentos empleamos 16 pun- tos para este cálculo basados en experimentación, utilizando diferente número de puntos, concluyendo que 16 puntos nos permiten reducir las altas frecuencias manteniendo el retardo en un mínimo aceptable para nuestro experimento.

La implementación del filtro en LabVIEW se realizó empleando un registro de desplazamiento que guarda un vector formado por la concatenación del valor actual con los valores anteriores del diámetro medido, el vector es recortado de acuerdo al tamaño del filtro.

Una vez determinado el vector se suman todos los valores y se divide el resultado entre el tamaño del filtro para obtener el valor de media móvil (figura 4). El valor obtenido se emplea entonces para calcular la diferencia entre el diámetro deseado y el promedio obtenido, y el resultado se emplea como entrada del algoritmo de control.

\section{Esquema en retroalimentación simple con un controlador neuronal auto-ajustable}

La figura 5 muestra el esquema general del control propuesto. Se muestran el filtro empleado para el diámetro medido, su comparación con el diámetro deseado, su alimentación a la red neuronal y la salida generada.
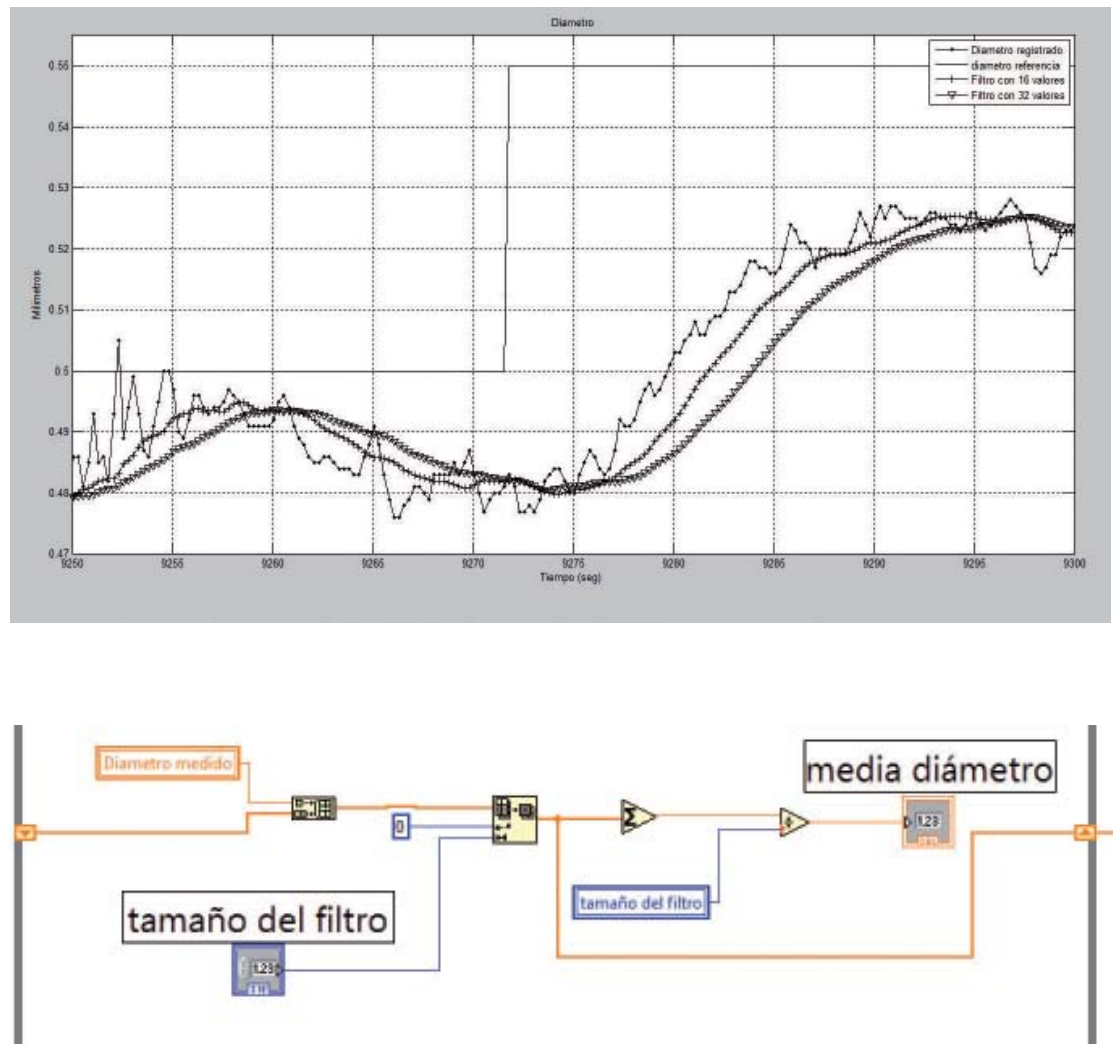

Figura 3. Desfase de la señal debido al tamaño del filtro

Figura 4. Implementación del filtro en LabVIEW 


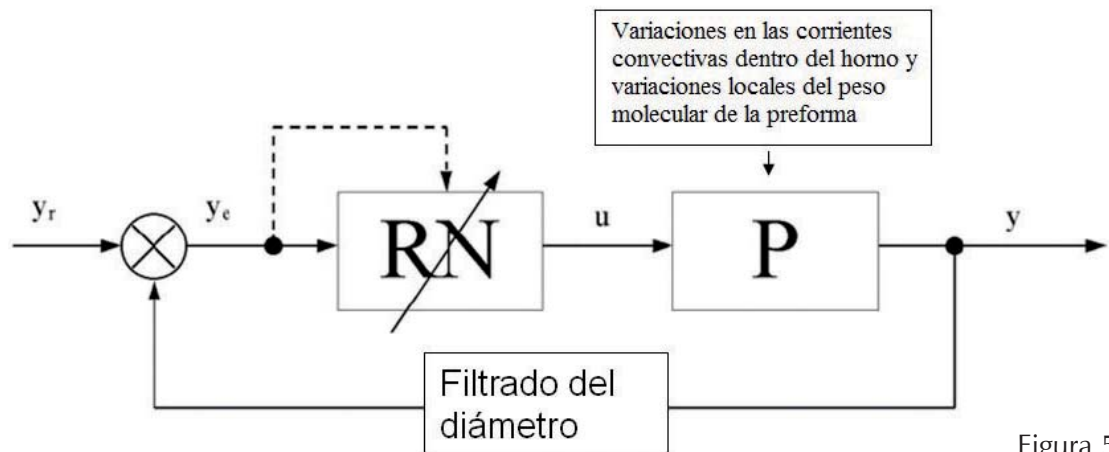

Figura 5. Esquema de control

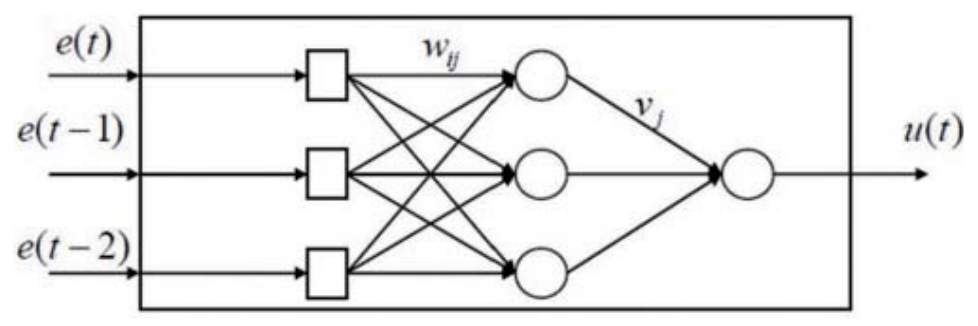

Figura 6. Estructura de red neuronal de control

La estructura de la red neuronal propuesta se muestra en la figura 6. En ésta se sustituye la etapa de aprendizaje por una adaptación continua en tiempo real de los coeficientes de peso, $w$ y $v$, de la red neuronal (Noriega et al., 2004)

La implementación de la red neuronal en LabVIEW se realizó mediante una plataforma MATLAB Script, que emplea un lenguaje similar al de MATLAB. El algoritmo desarrollado se incluye en la figura 7. Los valores de los pesos de los coeficientes $w_{i j}, v_{j}$, y la diferencia entre el diámetro de referencia (yr) y el diámetro medido (df) se introduce al control mediante registros de desplazamiento. El procedimiento de minimización consiste en un movimiento en la dirección negativa de la función $E(t)$ respecto a los coeficientes $w_{i j} \mathrm{y} v_{j}$ (Noriega et al., 2004).

$E[t]=\frac{1}{2} \sum_{k=1}^{t} e_{y}(k)^{2}$

El gradiente $E(t)$ es un vector multidimensional con componentes .

$\frac{\partial E(t)}{\partial v_{j}}, \frac{\partial E(t)}{\partial w_{i j}}$

La velocidad de modificación de los pesos está determinada por el valor del coeficiente de aprendizaje, alfa, que en los experimentos realizados se fijó en un valor de 0.8 .

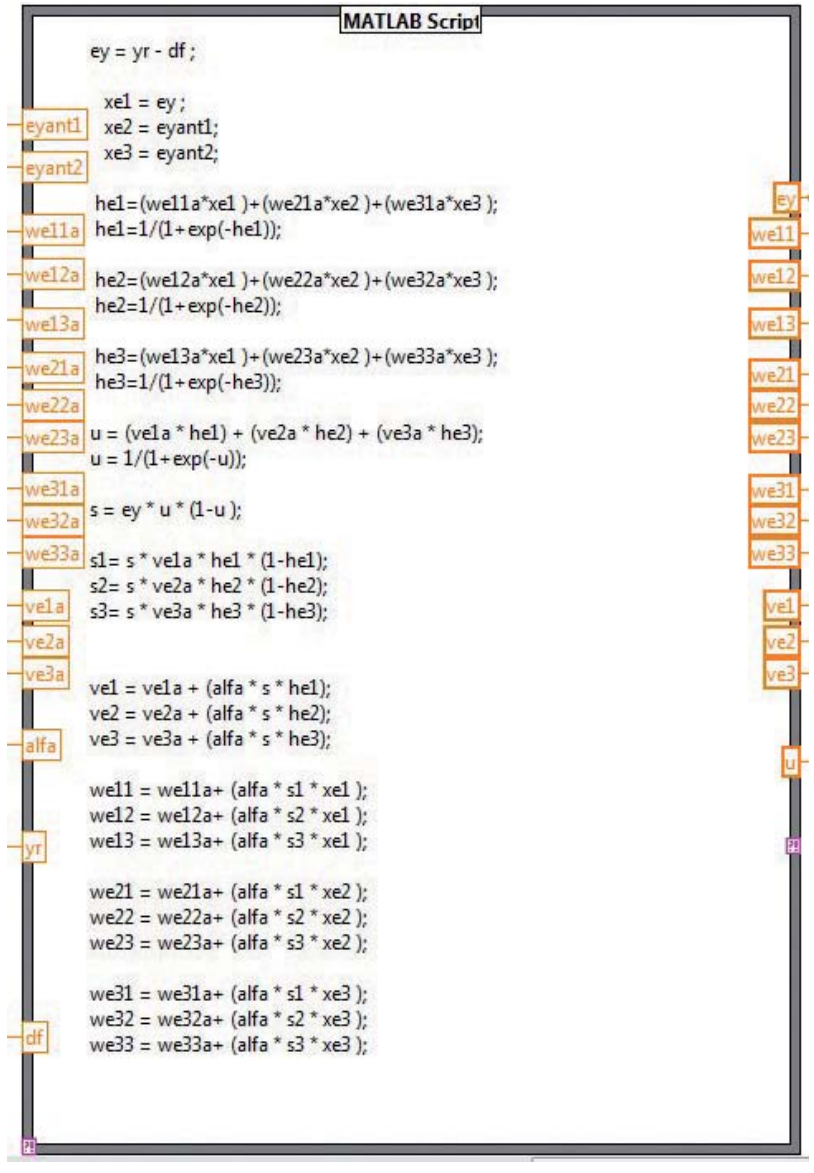

Figura 7. Implementación de la red neuronal en LabVIEW 


\section{Resultados}

Pruebas de estirado manual y automático

Los resultados de las pruebas se presentan en la siguiente tabla:

Tabla 1. Desviación estándar del diámetro medido y valor promedio (en $\mathrm{mm}$ ), para las operaciones con control automático y manual del sistema

\begin{tabular}{cccc}
\hline \multicolumn{5}{c}{ Prueba 1 } \\
\hline Desviación estándar & Media \\
Automático & 0.0046 & Automático & 0.5001 \\
Manual & 0.0163 & Manual & 0.4793 \\
\hline \multicolumn{5}{c}{ Prueba 2 } \\
\hline Desviación estándar & Media \\
Automático & 0.0025 & Automático & 0.5 \\
Manual & 0.0095 & Manual & 0.4923 \\
\hline \multicolumn{5}{c}{ Prueba 3 } \\
\hline Desviación estándar & Media \\
Automático & 0.0053 & Automático & 0.5005 \\
Manual & 0.0082 & Manual & 0.4922 \\
\hline \multicolumn{5}{c}{ Prueba 4 } \\
\hline Desviación estándar & Media \\
Automático & 0.0039 & Automático & 0.4997 \\
Manual & 0.0054 & Manual & 0.4892 \\
\hline \multicolumn{5}{c}{ Prueba 5 } \\
\hline Desviación estándar & 0.0068 & Automático & 0.4993 \\
Automático & 0.0104 & Manual & 0.4912 \\
Manual & \multicolumn{5}{c}{ Media } \\
\hline
\end{tabular}

En la figura 8 se muestran gráficamente los resultados de una de las pruebas realizadas. Se observa claramente que las variaciones de velocidad introducidas por la red neuronal mantienen los valores del diámetro registrado oscilando alrededor del valor deseado de 500 micras. Al pasar de control manual a automático y manteniendo la velocidad fija se observa una disminución en la desviación del diámetro que oscila con baja frecuencia alrededor del diámetro deseado manteniéndose las oscilaciones de alta frecuencia. Esto es consistente con la hipótesis de que las variaciones de diámetro de alta frecuencia se deben a las corrientes convectivas que se forman dentro del horno.

Un acercamiento a la zona de mayor variación de una de las pruebas durante el control automático, nos permite analizar la respuesta de velocidad del sistema de control. Los cambios de pendiente en la velocidad coinciden con el cruce de la variable controlada con el valor deseado; la velocidad de estirado aumenta progresivamente mientras la variable está por arriba del valor deseado y viceversa.

La figura 9 muestra una comparación de la variable de control (velocidad) contra la variable controlada (diámetro).
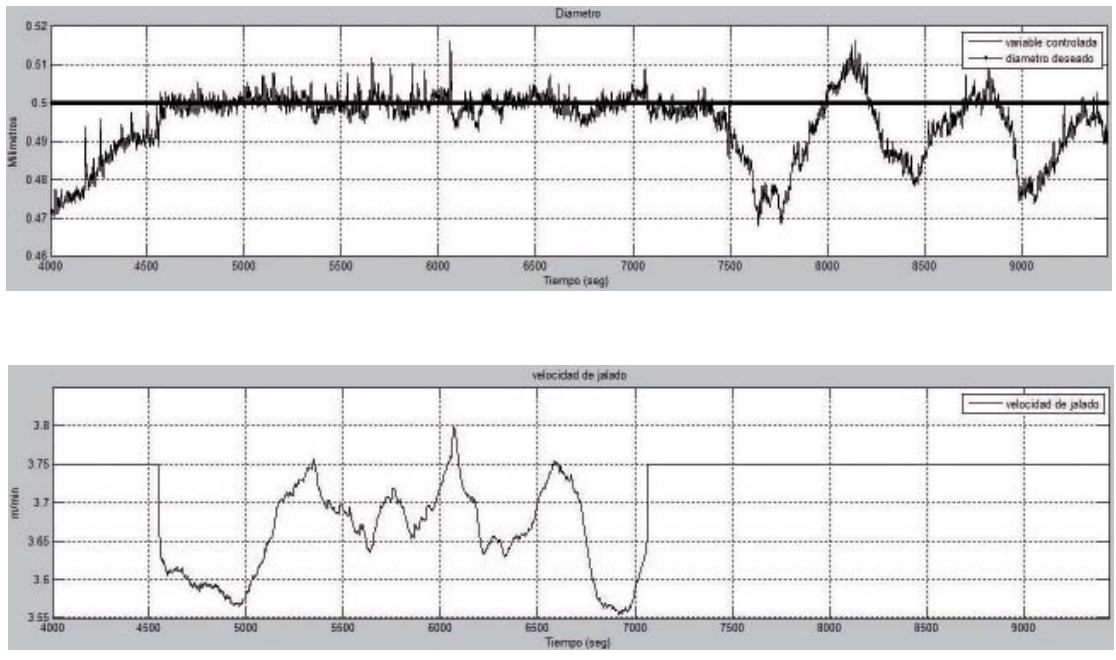

Figura 8. Gráfica obtenida de la segunda prueba 


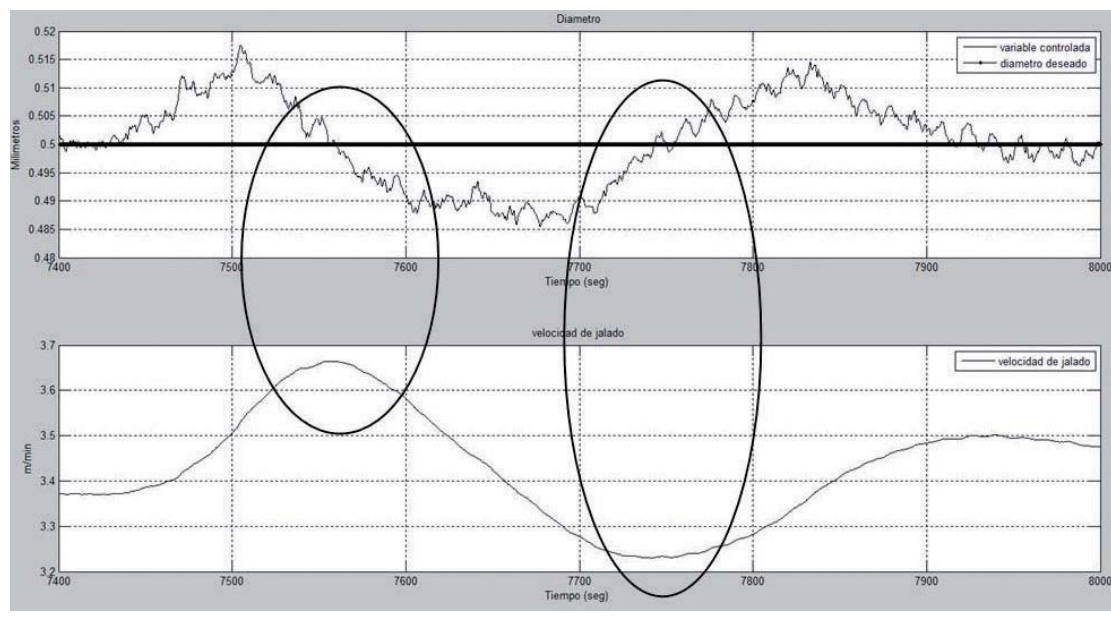

Figura 9. Comparación variable de control-variable controlada

\section{Conclusiones}

El algoritmo de control implementado en este trabajo se adapta a una variedad de procesos incluyendo los no lineales como es la producción de fibras ópticas. No es necesario un conocimiento a priori del modelo matemático del sistema para poder realizar un control, aunque si es necesario conocer cómo se comporta en general. Para determinar el valor apropiado del coeficiente de aprendizaje de la red sólo hacen falta unas cuantas pruebas y los coeficientes de los pesos $w_{i j}, v_{j}$ se adaptan en tiempo real de acuerdo a los cambios del sistema, lo cual permite que el control neuronal pueda ser usado en procesos con dinámicas difíciles. En el presente trabajo perturbaciones tales como las variaciones del peso molecular y variaciones longitudinales en el diámetro a lo largo de la preforma son compensados eficientemente por la red neuronal.

\section{Referencias}

Colmenares K.T., Suárez M.E., Uria M., Lamanna R. Implantación de un esquema de control PID autoajustable en un proceso químico modelado con redes neuronales. Revista de la Facultad de Ingeniería de la U.C.V., 16:107-114, 2001.

García A., Ocampo M.A., Saldívar-Guerra E., García-Gaitán B., Vivaldo-Lima E., Luna-Bárcenas G. Modified Frontal Polyme- rization of Poly (Methyl Methacrylate). Journal of Applied Polymer Science, 115:1289-1295, 2010.

Imoto K., Sumi M., Toda G., Suganuma T. Optical Fiber Drawing Method with Gas Flow Controlling System. J. Lightwave Technology, 7:115-121, 1989.

Lee K.M., Wei Z., Zhou Z., Hong S.P.,Computational Thermal Fluid Models for Design of a Modern Fiber Draw Process. IEEE Transactons on Automation Science and Engineering, 3:108118, 2006.

Nakahara M., Sakagauchi S., Miyashita T. Review of Electronic Communications Lab., 26:476-483, 1978.

Noriega-Ponce A., Aguado-Behar A., Ordaz-Hernández A., Rauch-Sitar V. Neural Networks for Self-tuning Control Systems, Czech Technical University in Prague. Acta Polytechnica, 44, 2004.

Reeve H.M., Mescher A.M. Effect of Unsteady Natural Convection on the Diameter of Drawn Polymer Optical Fiber. Optics Express, 11:1770-1779, 2003.

Reeve H.M., Mescher A.M., Emery A.F. Investigation of Convective Heating in a Polymer Fiber Drawing Process. Polymer Composites, 24:279-290, 2003.

Smith S.W. The Scientist and Engineer's Guide to Digital Signal Processing, 2a ed., Technical Publishing, California, 1999.

Valenzuela J.O. Obtención de fibras ópticas de poli (metil metacrilato) por el método de estirado. Tesis (maestría), México DF, Facultad de Química UNAM, 2006. 


\section{Semblanza de los autores}

Carlos Fuentes-Silva. Estudiante de la maestría en ciencias en la Universidad Autónoma de Querétaro, programa de instrumentación y control con especialidad en electrónica. Cursó la licenciatura en instrumentación y control en la UAQ. Su interés en investigación se centra en la producción de fibras ópticas poliméricas y desarrollo de instrumentos para la aplicación de las mismas, usando programación de alto y bajo nivel con software como LabVIEW y lenguaje VHDL en FPGA.

Alfonso Noriega-Ponce. Es ingeniero industrial en electricidad por el Instituto Tecnológico de Querétaro, México, egresado en 1977. Obtuvo el grado de maestría en ciencias de ingeniería eléctrica con especialidad en control automático en 1992 por el Centro de Investigación y de Estudios Avanzados del Instituto Politécnico Nacional en la Ciudad de México, DF. Desde 1986 a la fecha, es profesor de la Facultad de Ingeniería de la Universidad Autónoma de Querétaro, México. Sus áreas de interés son: control inteligente y control adaptable.

Miguel Ángel Ocampo-Mortera. Físico y maestro en ciencias por la Facultad de Ciencias de la UNAM, es doctor en ingeniería (materiales) por la Universidad Autónoma de Querétaro. Es responsable del Laboratorio de Fibras Ópticas del Centro de Física Aplicada y Tecnología Avanzada de la UNAM y miembro del SNI en el nivel I.

Ibrahim Serroukh. Doctor. Actualmente trabaja en la Facultad de Ingeniería de la Universidad Autónoma de Querétaro. 